\title{
Bestimmung der hyperkapnischen Atemantwort bei Patienten mit COPD
}

\author{
Determination of Hypercapnic Ventilatory Response in COPD Patients
}

Autoren

Institute
K. Sohrabi ${ }^{1}$, M. Scholtes ${ }^{1}$, L. Mursina ${ }^{1}$, A. Weissflog ${ }^{3}$, W. Hildebrandt ${ }^{4}$, O. Hildebrandt ${ }^{1,2}$, V. Gross ${ }^{1}$, U. Koehler ${ }^{2}$

Die Institutsangaben sind am Ende des Beitrags gelistet. eingereicht 27.5 .2015 akzeptiert nach Revision 5.8.2015

Bibliografie

Dol http://dx.doi.org/

10.1055/s-0034-1392998

Online-Publikation: 12.10.2015

Pneumologie 2015; 69: 662-666

(c) Georg Thieme Verlag KG

Stuttgart · New York

ISSN 0934-8387

Korrespondenzadresse

Prof. Dr. Keywan Sohrabi

Technische Hochschule

Mittelhessen

Fachbereich Gesundheit

Wiesenstraße 14

35390 Gießen

keywan.sohrabi@ges.thm.de

\section{Zusammenfassung \\ $\nabla$}

Die vorliegende Studie zeigt eine verringerte hyperkapnische Chemosensitivität (HCVR) bei COPD-Patienten. Bislang gibt es keine veröffentlichten Studien, die die HCVR im Bezug auf die COPD-GOLD-Stadien näher untersucht haben.

Die Messung der Atemantwort und des Atemantriebes auf Veränderungen der Blutgase stellen eine methodische Option dar, die Funktionsfähigkeit des „Regelkreises Atmung“ zu überprüfen. Zur automatischen und standardisierten Messung und Bewertung von hyperkapnischer Chemosensitivität und Atemantrieb wurde das MarburgerAtemantwort-Messungs-System (MATAM) entwickelt. Bei der Bestimmung der hyperkapnischen Atemantwort wird untersucht, ob eine $\mathrm{CO}_{2}$-Exposition zu einem adäquaten Anstieg von Atemfrequenz und Atemzugvolumen führt. Die Registrierungen werden in einem geschlossenen System, welches die selektive Veränderung jeweils nur einer Atemgas-Komponente ermöglicht, durchgeführt. Die Darstellung und Berechnung der hyperkapnischen Atemantwort erfolgt, indem das Atemminutenvolumen (AMV) zu Beginn der Reaktion gegen den endexspiratorischen $\mathrm{CO}_{2}\left(\mathrm{ETCO}_{2}\right)$ aufgetragen wird. Nachfolgend wird eine lineare Regressionsgerade berechnet, welche als Maß für die HCVR definiert ist.

Insgesamt wurden 28 Patienten (18 männliche; 10 weibliche) mit COPD-GOLD-Stadien 0-IV untersucht. Das Alter der Patienten lag im Mittel bei $57 \pm 14$ (Standardabweichung) und der BMI-Wert bei $32 \pm 9 \mathrm{~kg} / \mathrm{m}^{2}$. Es konnte gezeigt werden, dass die HCVR-Messung mittels MATAM bei COPDPatienten problemlos einsetzbar ist. Weiterhin konnte nachgewiesen werden, dass die hyperkapnische Atemantwort mit zunehmendem COPDSchweregrad signifikant abnimmt. Es wird vermutet, dass die permanente Verstellung der Blutparameter ( $\mathrm{pH}$ - und $\mathrm{pCO}_{2}$-Sättigung) zu einer Reduktion der Chemosensitivität führt. Es müssen

\section{Abstract \\ $\nabla$}

Previous studies showed a reduced hypercapnic ventilatory response (HCVR) in patients with COPD. However, the association between HCVR and COPD GOLD stages is unknown.

The measurement of the HCVR is a methodological option to test the function of the breathing feedback cycle. The aim of this feasibility study was to present a new automatic and standardized device (MATAM) to measure and interpret the HCVR. This device determines if exposure to $\mathrm{CO}_{2}$ leads to an adequate increase in breathing frequency and tidal volume. Recordings are performed in a closed system that allows selective changes of each gas component. The minute ventilation (AMV) under hypercapnic stimulation is plotted against the end-tidal $\mathrm{CO}_{2}\left(\mathrm{ETCO}_{2}\right)$. The HCVR is defined as the linear regression line.

28 patients (18 male; 10 female) with COPD GOLD stages 0 to IV were studied. The patients had a mean age of $57 \pm 14$ (standard deviation) years and a mean BMI of $32 \pm 9 \mathrm{~kg} / \mathrm{m}^{2}$. We could show that the HCVR measurement in patients with COPD using MATAM was feasible. Patients with more severe COPD stages had a significantly more reduced HCVR. This could be an indication of reduced chemosensitivity due to a worsening of blood values $\left(\mathrm{pH}\right.$ and $\mathrm{pCO}_{2}$ ) which affect the central chemoreceptors in the long term. Further studies will be needed to validate the MATAM device for healthy individuals and other patient groups, and for the investigation of standard values. 
nun weitere Studien mit diesem Verfahren erfolgen, um einerseits Normwerte zu ermitteln und andererseits das Verfahren auch für andere Patientengruppen zu validieren.

\section{Einleitung}

Die chemische Atmungsregulation passt die Ventilation den zellulären Stoffwechselbedürfnissen des Organismus an. Die Atmungsregulation erfolgt maßgeblich über periphere und zentrale Chemorezeptoren. Ein akuter Abfall des arteriellen Sauerstoffpartialdrucks $\left(\mathrm{paO}_{2}\right)$ bzw. ein Anstieg des Kohlendioxidpartialdrucks $\left(\mathrm{paCO}_{2}\right)$ bedingen eine Zunahme der Ventilation mit einer Erhöhung der Atemfrequenz sowie des Atemzugvolumens. Das Ansprechen der Chemorezeptoren auf Veränderungen der Blutgase wird als hypoxische oder hyperkapnische Atemantwort beschrieben. Die zentralen Chemorezeptoren reagieren insbesondere auf einen Anstieg des $\mathrm{paCO}_{2}$ bzw. Abfall des ph-Wertes im Liquor [1]. Bereits bei einer geringen Zunahme des $\mathrm{pCO}_{2}$ steigt die Ventilation deutlich an. Die im Vergleich träge Atmungsregulation über den $\mathrm{paO}_{2}$ beweist die dominierende Rolle des $\mathrm{pCO}_{2}$ als Mess- und Regelgröße in der Regulation der Atmung.

Die hyperkapnische Atemantwortmessung (HCVR = hypercapnic ventilatory response) wurde bislang mittels steady-state Verfahren nach Fenn und Craig [2 -4] oder nach der Rückatemmethode nach Read durchgeführt [5]. Es konnte bereits gezeigt werden, dass die Messung der HCVR insbesondere bei COPD-Patienten sinnvoll ist [6-8] und ein Maß für die Chemosensitivität darstellt $[9,10]$.

Heutzutage wird das methodisch einfacher praktizierbare und zeitlich kürzere Rückatmungsverfahren nach Read präferiert. Zur Quantifizierung der hyperkapnischen Atemantwort wird das Atemminutenvolumen als Funktion des petCO $\mathrm{C}_{2}$ dargestellt. Die Beziehung ist typischerweise linear, wobei die Steigung der Geraden das Maß der hyperkapnischen Atemantwort darstellt. Bei gesunden Versuchspersonen als auch bei Patienten besteht eine hohe Variabilität der hyperkapnischen Atemantwort. Hierfür dürften individuell prädisponierende Faktoren einerseits sowie andererseits auch methodische Probleme verantwortlich sein. Die Angewöhnungsphasen vor den Messungen dauern bei- spielsweise unterschiedlich lange, die individuellen Unterschiede im initialen $\mathrm{PetCO}_{2}$-Wert werden nicht adäquat berücksichtigt, die Sauerstoffkonzentration wird nicht stabil gehalten.

Im Rahmen dieser Arbeit soll ein neues automatisiertes Verfahren zur Bestimmung der hyperkapnischen Atemantwort (HCVR) bei Patienten mit COPD unterschiedlichen Schweregrades vorgestellt und evaluiert werden. Dabei sollen standardisierte Routinen in das System integriert werden, die zukünftig eine Vergleichbarkeit der Messparameter ermöglichen. Das Mess-, Steuerungs- und Regelungssystem soll dafür sorgen, dass die Gaskonzentrationen $\left(\mathrm{CO}_{2}\right.$ und $\left.\mathrm{O}_{2}\right)$ für den Patienten jederzeit einen definierten Zustand haben. Ein intelligenter Analysealgorithmus soll es zudem ermöglichen, den Punkt der signifikanten Ventilationssteigerung zu detektieren und nur die entsprechenden Werte in die Berechnung der Regressionsgeraden einzubeziehen.

\section{Material und Methode \\ $\nabla$}

\section{Patientenkollektiv}

Es wurden 28 COPD-Patienten (18 männlich und 10 weiblich) mit unterschiedlichem Schweregrad gemäß GOLD (The Global Initiative for Chronic Obstructive Lung Disease) untersucht. Dabei waren sechs Patienten dem Stadium 0-I, zwölf dem Stadium II, sechs dem Stadium III und vier dem Stadium IV zugeordnet. Die Patienten hatten einen mittleren BMI von $32 \pm 9$ (Mittelwert \pm Standardabweichung) und ein durchschnittliches Alter von $57 \pm 14$ Jahren.

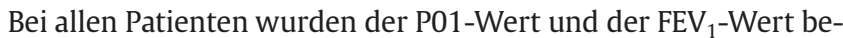
stimmt und eine entsprechende Einteilung in COPD-Schweregrade nach GOLD vorgenommen. Anschließend wurde bei allen Patienten die HCVR mittels Marburger-Atemantwort-Messung (MATAM) bestimmt. Die Studie wurde von der lokalen Ethikkommission genehmigt (Ethikvotum: Studie 37/05).

\section{Die Marburger-Atemantwort-Messung}

Im Rahmen dieser Studie wurde ein Gerät entwickelt, das eine automatische Bestimmung der HCVR ermöglicht ( $\bullet$ Abb.1). Mit Hilfe dieser neuen Methode kann der Proband/Patient bei verschlossener Nasenöffnung über ein Mundstück mit einem geschlossenen Gas-Kreislauf verbunden werden. Hier können defi-

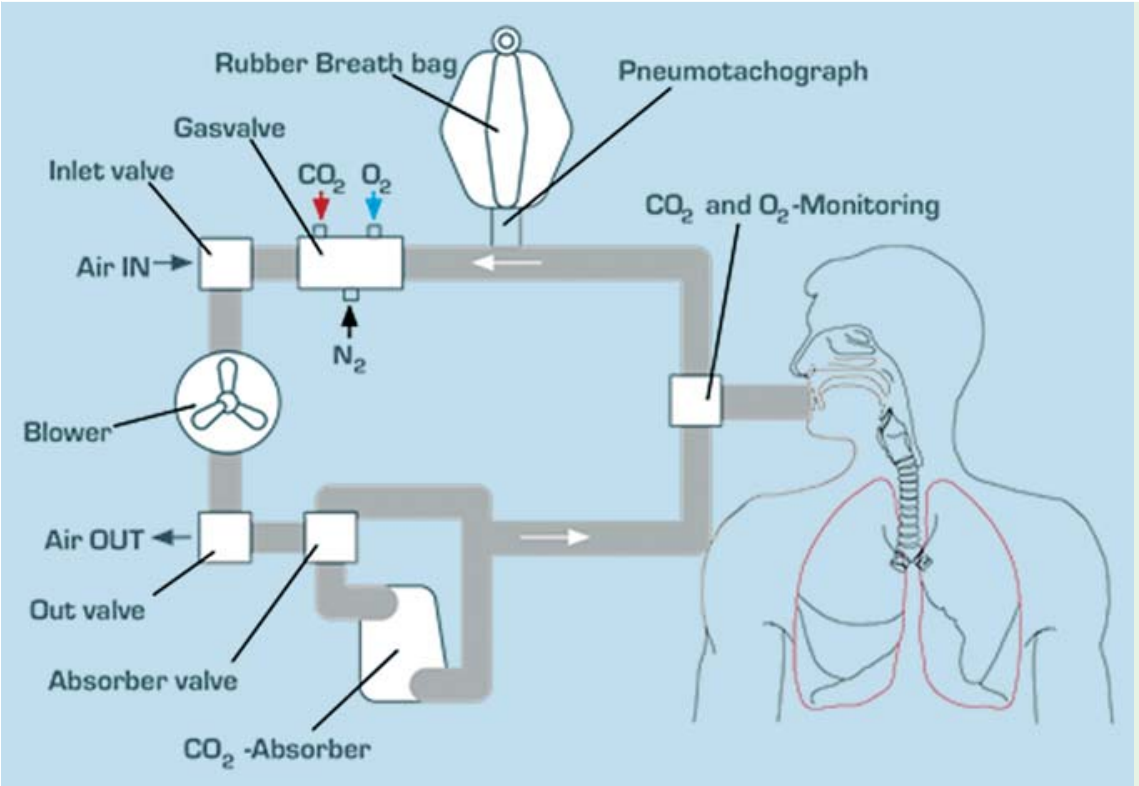

Abb. 1 Schematische Darstellung der MarburgerAtemantwort-Messung. 
nierte hyperkapnische Gasgemische erzeugt werden. Über computergesteuerte Einlassventile können einerseits gezielt aus $\mathrm{O}_{2-}$, $\mathrm{CO}_{2}$ - und $\mathrm{N}_{2}$-Gasflaschen die entsprechenden Gase in das System geleitet werden, andererseits können auch die $\mathrm{O}_{2}$ - und $\mathrm{CO}_{2}$-Konzentrationen durch variable Rückatmung beeinflusst werden. Bei der Messung der hyperkapnischen Atemantwort besteht die Schwierigkeit allgemein darin, abzugrenzen, ob die gemessene Atemantwort den physiologischen Bedingungen $\left(\mathrm{PO}_{2}, \mathrm{PCO}_{2}\right.$ oder des pH-Wertes) zugrunde liegt oder ob sie durch das Messverfahren zusätzlich beeinflusst wird. Um dieser Problematik entgegenzuwirken wurde das MATAM-Verfahren so entwickelt, dass eine automatische regulierbare isokapnische (kontrollierte $\mathrm{CO}_{2}$ ) oder isooxische (kontrollierte $\mathrm{O}_{2}$ ) Bedingung gewährleistet wird. Dadurch werden während der gesamten Phase des Provokationstestes gleichbleibende Voraussetzungen geschaffen. Für die im Rahmen der vorliegenden Arbeit erfassten HCVR-Test-Ergebnisse bedeutet dies, dass während der Provokationstests die $\mathrm{O}_{2}$-Komponente stets konstant bei $40 \mathrm{Vol} \%$ gehalten und somit eine Manipulation durch den Sauerstoffgehalt ausgeschlossen wird.

Über Druck- und Gassensoren werden der Momentanzustand des Systems sowie verschiedene atemphysiologische Parameter simultan angezeigt und gespeichert. Die Messung von $\mathrm{O}_{2}$ und $\mathrm{CO}_{2}$ erfolgt mit Hilfe des Atemgasmonitors Capnomac Ultima (ULT-S) (Datex Engstrom Division, Finnland). Dabei wird über einen Probenschlauch kontinuierlich eine Gasprobe am Mundstück entnommen und in einer Messkammer spektroskopisch ausgewertet. Mit Hilfe des Ohr-Pulsoximeters Capnomac Ultima (ULT-S) (Datex Engstrom Division, Finland) werden die kapillare Sauerstoffsättigung $\left(\mathrm{SpO}_{2}\right)$ sowie die Pulsfrequenz (HF) gemessen. Atemfrequenz (AF) und Atemzugvolumen (AZV) werden mit dem Pneumotachografen A. Fleisch, Model 2 (Jäger, Deutschland) registriert. Das errechnete Atemminutenvolumen (AMV) wird für die Auswertung gegen $\mathrm{PetCO}_{2}$ aufgetragen.

Der systeminhärente Messablauf ist mehrstufig ( $\bullet$ Abb.2). Zunächst wird der Proband an das System gewöhnt, dabei atmet er durch das Mundstück in das offene System. In dieser Phase werden die Ruhewerte für $\mathrm{PetCO}_{2}, \mathrm{AMV}, \mathrm{SpO}_{2}$ und $\mathrm{HF}$ ermittelt. In der nächsten Phase wird der Patient vom System abgekoppelt, um intern das initiale Gasgemisch zu erzeugen. Dabei wird der mittlere $\mathrm{PetCO}_{2}$ aus der ersten Phase als initialer $\mathrm{CO}_{2}$-Gehalt für die Provokationsphase vorgemischt. Hierdurch wird sicherge-

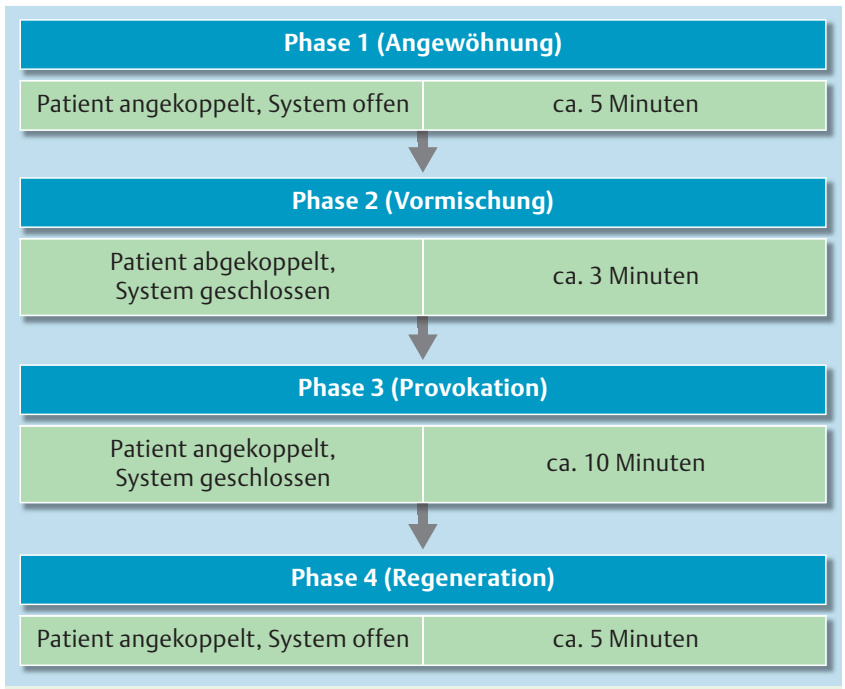

Abb.2 Die vier Phasen der MATAM-Messungen.

stellt, dass jeder Patient die Messung mit einer individuell angepassten Gaskonzentration startet. Diese setzt sich wie folgt zusammen: Der $\mathrm{CO}_{2}$-Gehalt entspricht dem vorher ermittelten mittleren $\mathrm{PetCO}_{2}$ des Patienten, der $\mathrm{O}_{2}$-Gehalt liegt bei $21 \mathrm{Vol} . \%$, und der Rest wird mit $\mathrm{N}_{2}$ aufgefüllt. In der dritten Phase wird der Patient wieder an das System angekoppelt, atmet aus dem individuell angepassten Gemisch und erhöht die $\mathrm{CO}_{2}$-Konzentration im System durch Rückatmung. In dieser Phase kommt es zu der eigentlichen Provokationsmessung und damit zur Bestimmung der HCVR. Das Gasgemisch bleibt dabei isooxisch bei stetig steigenden $\mathrm{CO}_{2}$-Konzentrationen. In der vierten und letzten Phase wird das System wieder geöffnet (Frischluft Flutung), um das Regenerationsverhalten des Patienten beobachten zu können.

Die Analyse der Provokationsmessung erfolgt zweistufig, entsprechend der schematischen Darstellung in $\bigcirc$ Abb. 3. Zunächst wird der Punkt Pet $\mathrm{CO}_{2}$-Wert gesucht, bei dem eine signifikante Steigerung des AMV auftritt. Ab diesem Punkt beginnt die physiologische Reaktion auf den $\mathrm{CO}_{2}$-Reiz. Im zweiten Schritt wird die Steigung der Regressionsgeraden für diese Werte ermittelt die HCVR.

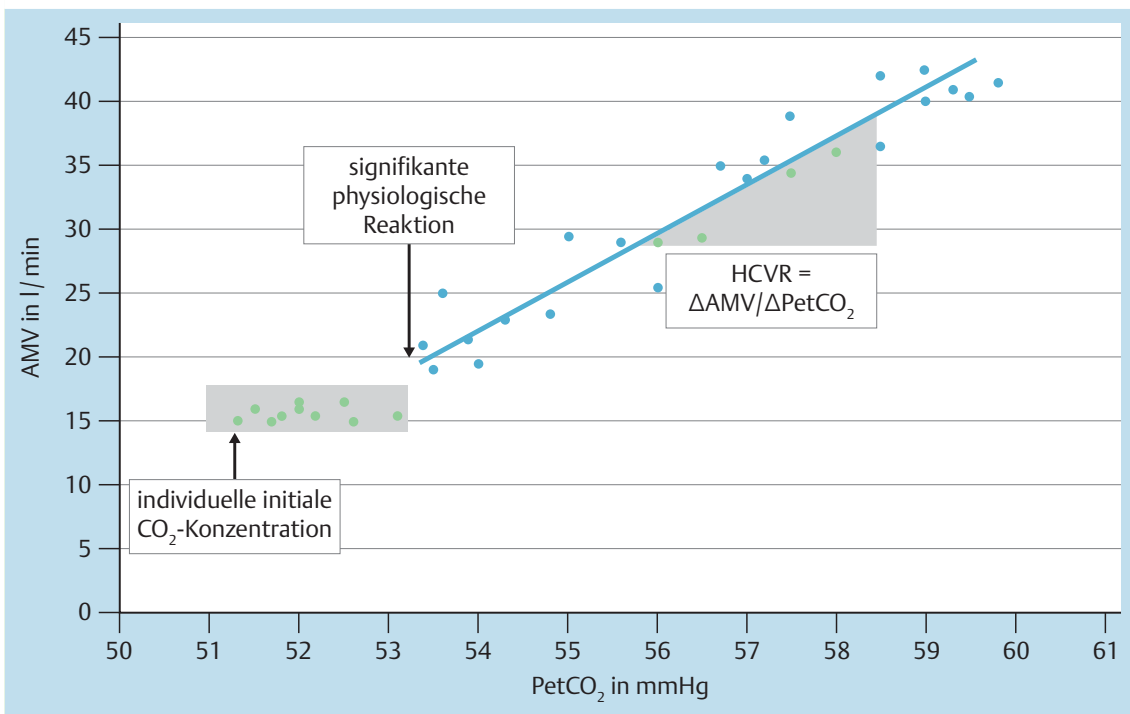

Abb.3 Zweistufige Bestimmung der hyperkapnischen Atemantwort mit dem standardisierten MATAM-Verfahren. 


\section{Statistik}

Die statistische Auswertung erfolgte mittels IBM SPSS Statistics Version 22 (IBM Deutschland GmbH, IBM-Allee 1, 71139 Ehningen). Die Normalverteilung der HCVR-Daten konnte mittels Sichtprüfung des Histogramms ausgeschlossen werden. Daher wurde ein nicht-parametrischer Test (Rangvarianzanalyse für unabhängige Stichproben nach Kruskal-Wallis) gerechnet. Für einen paarweisen Vergleich der einzelnen Schweregrade untereinander wurden entsprechende Post-Hoc-Analysen (U-Test nach Mann-Whitney) durchgeführt. Um einen Zusammenhang zwischen dem COPD-Schweregrad und der HCVR zu ermitteln, wurde der Rangkorrelationskoeffizient nach Spearman berechnet. Subanalysen für das Aufzeigen von geschlechtsspezifischen Unterschieden im Kollektiv wurden mittels zweiseitigem t-Test für unabhängige Stichproben durchgeführt.

\section{Ergebnisse}

Die Ergebnisse der vorliegenden Studie basieren auf den Untersuchungen der HCVR bei 28 COPD-Patienten mit unterschiedlichem Schweregrad. Als zentraler Befund konnte eine signifikante $(p=0,005)$ Verminderung der HCVR bei erhöhtem COPD-Schweregrad festgestellt werden ( $\bullet$ Abb.4). Beim paarweisen Vergleich ergaben sich zwischen "Schweregrad 4“ und "Schweregrade 0 \& 1 “ $(p=0,01)$ sowie zwischen „Schweregrad 4“ und „Schweregrad 2“ $(p=0,033)$ signifikante Unterschiede. Diese Ergebnisse bestätigten sich durch eine signifikante und hohe negative Korrelation von $r=-0,672(p<0,001)$ zwischen den HCVR-Werten und den jeweiligen Schweregradstadien.

Die Messung der P01-Werte, als Prädiktor für die Atemmuskelkraft, ergab keinen signifikanten Unterschied zwischen den COPD-Schweregraden nach GOLD ( $\bullet$ Abb.5).

Es gab weder signifikante Unterschiede zwischen weiblichen und männlichen Patienten in der Verteilung über die Schweregrade, beim Alter und Body Mass Index (BMI) noch bei den ermittelten Werten für HCVR und $\mathrm{FEV}_{1}(\bullet$ Tab.1). Das Patientenkollektiv zeigte keine geschlechtsspezifischen Auffälligkeiten.

\section{Diskussion}

\section{Evaluation der Methode}

Es konnte nachgewiesen werden, dass die hyperkapnische Atemantwort mit zunehmendem COPD-Schweregrad signifikant abnimmt. Das Messverfahren war auch bei respiratorisch stark eingeschränkten COPD-Patienten (mit hohem Schweregrad) anwendbar. Das MATAM-Verfahren konnte bei COPD-Patienten problemlos eingesetzt werden und stärkt damit die Aussagen von Nickol et al. [11], dass die Rückatemmethode für COPD-Pa-

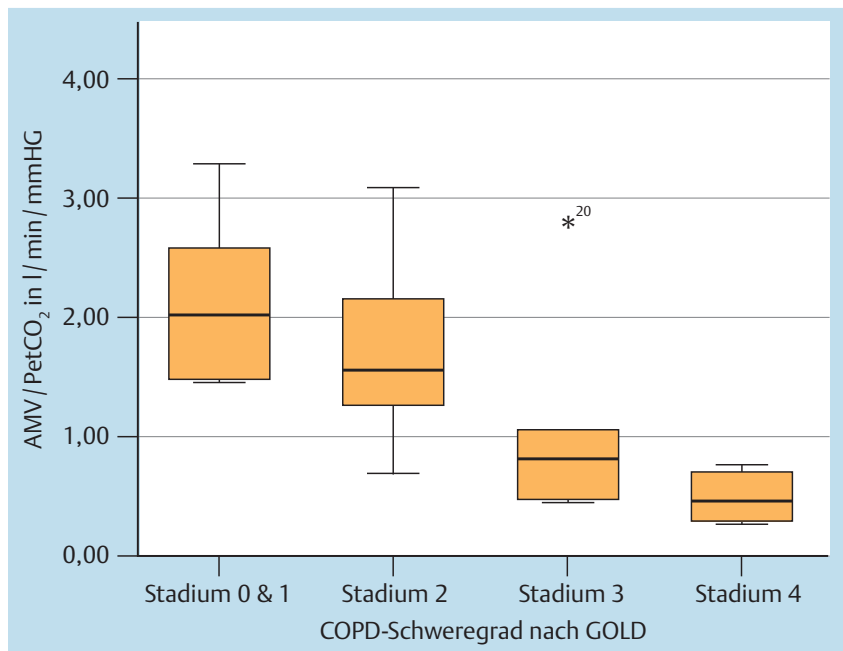

Abb.4 Die Verteilung der HCVR für die jeweiligen COPD-Schweregrade nach GOLD.

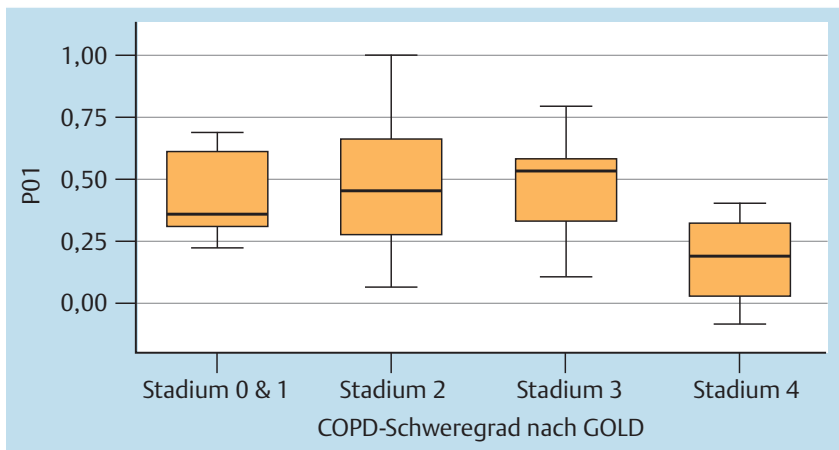

Abb.5 Die Verteilung der P01-Werte für die jeweiligen COPD-Schweregrade nach GOLD.

tienten geeignet ist. Die Ergebnisse zeigen einen deutlichen $\mathrm{Zu}$ sammenhang zwischen dem AMV und dem COPD-Schweregrad nach GOLD und damit dem $\mathrm{FEV}_{1}(\bullet$ Abb.4). Die Einflussfaktoren für die verminderte Atemantwort könnten sowohl an einer Rezeptorverstellung als auch an einer Schwächung der Muskelkraft liegen. Da die P01-Werte über die Schweregrade der COPD keine signifikanten Unterschiede aufwiesen, vermuten wir, dass es sich um eine Rezeptorverstellung handelt. In zukünftigen Studien könnte diese Frage durch die zusätzliche Erhebung von Blutgaswerten und Muskelbiopsien genauer untersucht werden.

Da während der Provokationsmessung AF und AZV bestimmt werden, könnten auch diese getrennt voneinander über PetCO aufgetragen werden, um einen Eindruck zu gewinnen, ob die

Tab. 1 Ergebnisse der t-Test Analysen für geschlechtsspezifische Unterschiede.

\begin{tabular}{|c|c|c|c|c|c|}
\hline & \multicolumn{2}{|l|}{ Weiblich } & \multicolumn{3}{|l|}{ Männlich } \\
\hline & Mittelwert & Standardabweichung & Mittelwert & Standardabweichung & p-Wert \\
\hline Alter (Jahre) & 58,3 & 17,1 & 57,2 & 13,7 & 0,85 \\
\hline BMI & 32,5 & 10,4 & 32,2 & 8,6 & 0,94 \\
\hline HCVR $(1 / \mathrm{min} / \mathrm{mmHg})$ & 1,2 & 0,7 & 1,6 & 0,9 & 0,25 \\
\hline $\mathrm{FEV}_{1}(\%)$ & 63,2 & 28,6 & 60,3 & 22,9 & 0,77 \\
\hline Schweregrad (GOLD) & 2,3 & 1,1 & 2,2 & 0,8 & 0,95 \\
\hline
\end{tabular}


physiologische Reaktion eher über eine Steigerung der AF oder des AZV erfolgt. Diese differenzierte Analyse könnte möglicherweise bereits in frühen COPD-Stadien Hinweise darauf geben, ob ein Patient eher zur phänotypischen Ausprägung „Pink Puffer“ oder „Blue Bloater“ neigt. Der „Pink Puffer“ neigt dazu, seine Ventilation durch die Zunahme der AF zu steigern, wohingegen der „Blue Bloater“ eher das AZV erhöht. Damit ist MATAM ein geeignetes und standardisiertes Verfahren zur Bestimmung der HCVR bei COPD-Patienten und könnte als Medizinprodukt etabliert werden.

\section{Limitationen}

Die Machbarkeitsuntersuchung erfolgte nur an COPD-Patienten und muss nun auch an lungengesunden Probanden sowie anderen Patientenkollektiven vorgenommen werden. Es wurde nur der $\mathrm{PetCO}_{2}$ als Stellvertreter für $\mathrm{PaCO}_{2}$ gemessen, sodass keine Aussagen über den tatsächlichen $\mathrm{PaCO}_{2}$ getroffen werden können. Allerdings lässt sich vermuten, dass zumindest eine proportionale Abhängigkeit zwischen beiden Werten besteht und somit eine qualitative Aussage über den Steigerungsgrad möglich ist.

\section{Ausblick}

Die von uns entwickelte MATAM-Methode hat gezeigt, dass eine automatische und standardisierte Bestimmung der Atemantwort möglich ist. Unser Verfahren eröffnet damit neue und weitreichende diagnostische Optionen für den klinischen Alltag. Die Methode könnte, nicht zuletzt auf Grund der zentralen Funktion der hyperkapnischen Chemosensitivität im Regulationskreislauf der Atmung, zukünftig diagnostisch und therapeutisch kontrollierend an Bedeutung gewinnen. Im Rahmen der Weiterentwicklung wird der Auswertealgorithmus zusätzlich die Daten aus Phase 4 (Regeneration) als standardisierten Parameter für die Regenerationsfähigkeit der Patienten ausgeben. Hier müssen zunächst weitere Untersuchungen durchgeführt werden, um die Bedeutung dieser Phase einschätzen zu können. Da es sich um ein mitarbeitsunabhängiges Verfahren handelt, kann die Bestimmung der HCVR insbesondere im Bereich der Arbeitsmedizin zur Bestimmung von Chemosensitivitäts-Störungen wie bei COPDPatienten unterstützend eingesetzt werden. Es müssen nun weitere Studien mit diesem Verfahren erfolgen, um einerseits Normwerte zu ermitteln und das Verfahren auch für andere Patienten zu validieren. Dazu wäre es sinnvoll, dieses schnelle und einfache sowie standardisierte Verfahren als zugelassenes Medizinprodukt multizentrisch einzusetzen.

\section{Danksagung}

Wir danken allen Patientinnen und Patienten, die sich bereit erklärt haben, uns bei dieser Studie zu unterstützen. Dieses Projekt (HA-Projekt-Nr.: 138/07-01) wurde im Rahmen der Innovationsförderung des Landes Hessen gefördert, kofinanziert aus Mitteln der Europäischen Union (Europäischer Fonds für Regionale Entwicklung - EFRE).

\section{Interessenkonflikt}

Prof. Dr. U. Koehler: Heinen und Löwenstein, IfM GmbH, GlaxoSmithKline, Resmed, UCB Biosciences, Boehringer Ingelheim.

Prof. Dr. V. Groß: Heinen und Löwenstein.

Die anderen Autoren geben an, dass kein Interessenkonflikt besteht.

\section{Institute}

${ }^{1}$ Technische Hochschule Mittelhessen, Gießen, Fachbereich Gesundheit

2 Klinik für Innere Medizin, SP Pneumologie, Intensiv- und Schlafmedizin

(Direktor: Prof. Dr. C. Vogelmeier), Philipps-Universität Marburg

3 ThoraTech GmbH, Gießen

${ }^{4}$ Institut für Anatomie und Zellbiologie, Philipps-Universität Marburg

\section{Literatur}

1 Guyenet PG, Stornetta RL, Bayliss DA. Central respiratory chemoreception. J Comp Neurol 2010; 518: 3883-3906

2 Fenn WO, Craig AB. Effect of $\mathrm{CO} 2$ on respiration using a new method of administering CO2. J Appl Physiol 1963; 18: 1023-1024

3 McConnell AK, Semple ES. Ventilatory sensitivity to carbon dioxide: the influence of exercise and athleticism. Med Sci Sports Exerc 1996; 28: 685-691

4 Warley A, Clarke M, Phillips $T$ et al. Ventilatory response to an inhaled constant CO2 load and added dead space in healthy men, awake and asleep. Respir Physiol 1989; 75: 183-191

5 Read DJ. A clinical method for assessing the ventilatory response to carbon dioxide. Australas Ann Med 1967; 16: 20-32

6 Elliott MW, Mulvey DA, Moxham J et al. Domiciliary nocturnal nasal intermittent positive pressure ventilation in COPD: mechanisms underlying changes in arterial blood gas tensions. Eur Respir J 1991; 4: $1044-1052$

7 Nickol AH, Hart N, Hopkinson NS et al. Mechanisms of improvement of respiratory failure in patients with restrictive thoracic disease treated with non-invasive ventilation. Thorax 2005; 60: 754-760

8 Nickol AH, Hart N, Hopkinson NS et al. Mechanisms of improvement of respiratory failure in patients with COPD treated with NIV. Int J Chron Obstruct Pulmon Dis 2008; 3: 453-462

9 Browne HAK, Adams L, Simonds AK et al. Ageing does not influence the sleep-related decrease in the hypercapnic ventilatory response. Eur Respir J 2003; 21: 523-529

10 Filley GF, Swanson GD, Kindig NB. Chemical breathing controls: slow, intermediate and fast. Clin Chest Med 1980; 1: 13-32

11 Nickol AH, Dunroy H, Polkey MI et al. A quick and easy method of measuring the hypercapnic ventilatory response in patients with COPD. Respir Med 2009; 103: 258-267 